\title{
Prótesis removible en el paciente geriátrico
}

\section{Removable prosthesis in the geriatric patient}

\author{
Ayuso-Montero R*, Martori López E*, Brufau de Barberá $M^{* *}$, Ribera Uribe $M^{* * *}$
}

\section{RESUMEN}

En nuestro entorno sociodemográfico, según las condiciones económicas actuales, cada vez más pacientes ancianos optan por rehabilitar su boca mediante prótesis removible, y aún siendo las prótesis parciales removibles y prótesis completas tratamientos estudiados y descritos desde hace mucho tiempo, todavía hoy es básico el conocimiento de las técnicas y pasos para su elaboración. Además, son la base sobre la que se construyen las rehabilitaciones removibles sobre implantes, que ayudan a muchos pacientes geriátricos con problemas de retención y estabilidad en su prótesis removible. En el artículo se resume la situación actual de las prótesis removibles parciales, completas y sobre implantes, las técnicas clásicas de confección y las novedades más actuales en cuanto a materiales, diseño y elaboración por CAD-CAM, y se describe la importancia del conocimiento por parte del paciente y sus cuidadores de las instrucciones de higiene y mantenimiento para garantizar buenos resultados a largo plazo.

Palabras clave: Prótesis completa, prótesis parcial removible, sobredentadura, paciente mayor.

\section{SUMMARY}

Actually, in our sociodemographic environment, and according to the economic conditions, more and more elderly patients use removable prostheses, and being removable partial dentures and complete dentures, treatments studied and described long ago, the knowledge of techniques and steps for developing this type of prosthesis remain basic yet today. In addition, it is also the basis for the removable restorations on implants, which help many elderly with retention and stability requirements in their removable dentures. The article summarize the current situation of partial, complete and implant dentures, classical confection techniques and the latest developments in materials, design and fabrication by CAD-CAM, and describes the importance of the patient and caregivers knowledge for a correct hygiene and maintenance to ensure long-term success.

Key words: Complete denture, partial removable denture, overdenture, elderly patient.

Fecha de recepción: 2 de mayo 2015.

Aceptado para publicación: 6 de mayo 2015.

Ayuso-Montero R, Martori López E, Brufau de Barberá M, Ribera Uribe M. Prótesis removible en el paciente geriátrico. Av. Odontoestomatol 2015; 31 (3): 191-201.

* $\quad$ Facultad de Odontología. Universidad de Barcelona.

** Facultad de Odontología. Universidad Internacional de Cataluña.

\section{INTRODUCCIÓN}

El impacto demográfico, con el aumento de la longevidad, y la actual crisis económica están suponiendo un acicate para la utilización de técnicas de rehabilitación oral, como la prótesis parcial removi- ble, que clásicamente había sido relegada a un segundo término en pro de tratamientos más onerosos pero de mayor funcionalidad y estética, como son las prótesis fijas sobre dientes naturales o sobre implantes. Estamos viendo cómo un número creciente de pacientes ancianos buscan soluciones a su 
edentulismo recurriendo a las prótesis removibles convencionales (Fig. 1). En este sentido el diseño y elaboración de este tipo de prótesis puede y debe llevarse a cabo con la misma atención y excelencia que cualquier otra de las soluciones más costosas. De hecho si nos atenemos a las necesidades y demandas reales de los pacientes podemos ver que en torno a un $12,8 \%$ de la población entre 65 y 74 años es portadora de una prótesis parcial removible superior y un 16,7 \% inferior, según la encuesta de salud oral del 2010.

La protocolización en la elaboración de las prótesis es indispensable para obtener un estándar de calidad, prever incidencias futuras, acortar los tiempos de tratamiento y disminuir los costes, aspectos todos ellos que harán más predecible el resultado final. Realmente todo ello es imprescindible en pacientes de cualquier edad, pero de modo más significativo en pacientes ancianos para los que el coste, las incidencias y los desplazamientos suponen un hándicap mucho mayor.

Es importante desde la primera cita definir con el paciente o con su cuidador dos aspectos básicos:

1. La necesidad de higiene y mantenimiento de prótesis y dientes remanentes, $y$

2. Las ventajas e inconvenientes de la prótesis parcial removible contrastándolas con las expectativas de pacientes y cuidadores.

El paciente y sus familiares deben ser conscientes de que, adjunto a la rehabilitación y de manera indivisi-

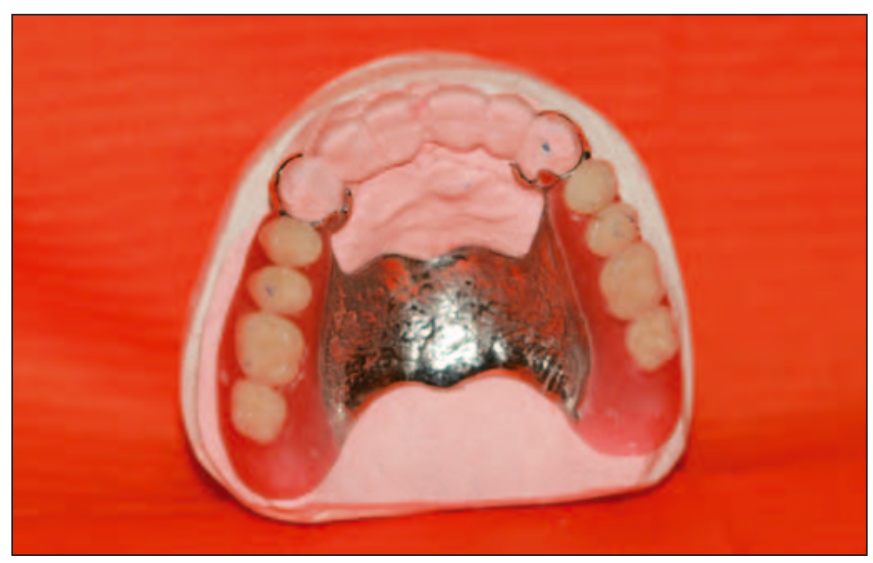

Fig. 1. Prótesis parcial removible convencional. ble, se le va a indicar un plan de mantenimiento que es de capital importancia en el éxito del tratamiento a largo plazo. Mantenimiento que hemos de encargaremos de detallar el primer día de visita, indicando que será responsabilidad nuestra cuenta el recordarle las citas necesarias.

\section{PRÓTESIS CONVENCIONAL}

En esta primera toma de contacto a la que el paciente debe acudir con todos los tratamientos quirúrgicos o restauradores efectuados y obviamente con la historia clínica cumplimentada, tomaremos unos registros o modelos de estudio mediante impresiones con hidrocoloides irreversibles y unas relaciones intermaxilares. Para ello, cuando hay estabilidad oclusal o arcadas dentosoportadas como las clases III y IV de Kennedy, se toma una cera en máxima intercuspidación y cuando no la hay o en las clases I, II y $\mathrm{V}$ de Kennedy, lo que se toma es un registro intermaxilar de silicona que permita la fabricación previa de un rodete de resina sobre el cual registrar también las relaciones intermaxilares con cera. De esta manera podremos obtener, mediante el uso del arco facial, el montaje del caso en articulador semiajustable. Para ello, en el laboratorio se procederá al vaciado, zocalado y montaje en articulador de los modelos. Posteriormente se efectuará el análisis y diseño de los elementos de la prótesis mediante el uso del paralelizador (Fig. 2). En los últimos tiempos y al amparo de la pujanza de la utilización de técnicas de diseño informatizadas y sistemas CAD CAM, se ha propuesto la realización del diseño de la estructura metálica de las prótesis removibles mediante softwares específicos y captación de la imagen de los modelos (1). No hay evidencias científicas de que en el estado actual de la técnica, el uso de CAD CAM en el diseño de las prótesis removibles suponga una ventaja respecto de las técnicas convencionales, aunque es un interesante campo para futuras investigaciones.

El diseño es una pieza clave del proceso de elaboración. Forma parte de las competencias y obligaciones del dentista y tiene en cuenta variables que tan solo él conoce y cuya valoración no puede delegar en el técnico de laboratorio. Es especialmente notorio a la hora de diseñar: 


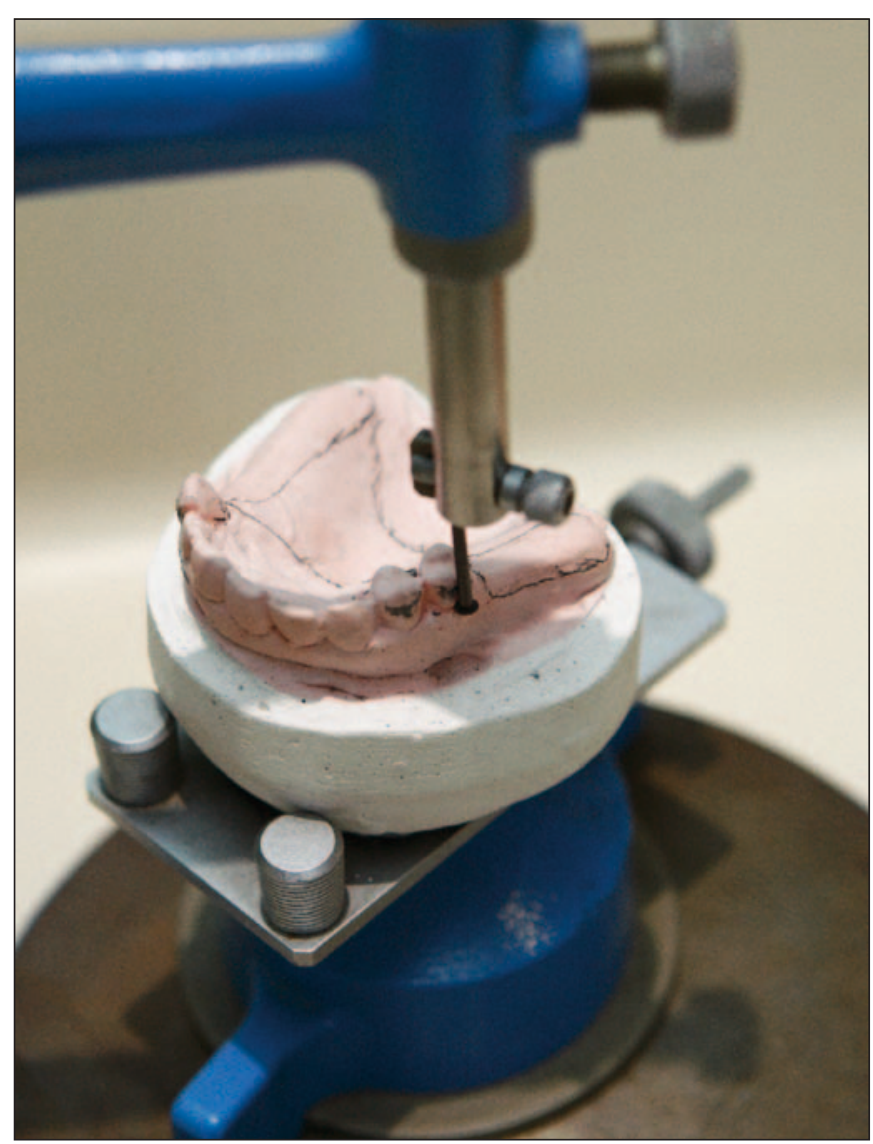

Fig. 2. Análisis del diseño mediante paralelizador.

1. La necesidad de definir ejes de inserción que permitan al anciano la fácil remoción e inserción de la prótesis en boca teniendo en cuenta las dificultades de movilidad, las patologías limitantes como artritis y las limitaciones tempo-espaciales propias de la edad.

2. La importancia de tener en cuenta que la pérdida de posibles dientes, especialmente dientes pilares, no suponga una alteración biomecánica importante en el diseño, un sobrecoste excesivo o una reparación que se dilate en el tiempo.

3. El que los elementos que forman parte de la estructura, como conectores mayores o menores, retenedores indirectos, etc., sean de diseño simple, permitan la higiene y respeten al máximo la salud periodontal facilitando la autoclisis (Fig. 3).

Del mismo modo, durante el diseño se deben tener en cuenta las consideraciones estéticas para incorporar modificaciones dentales y materiales apropia-

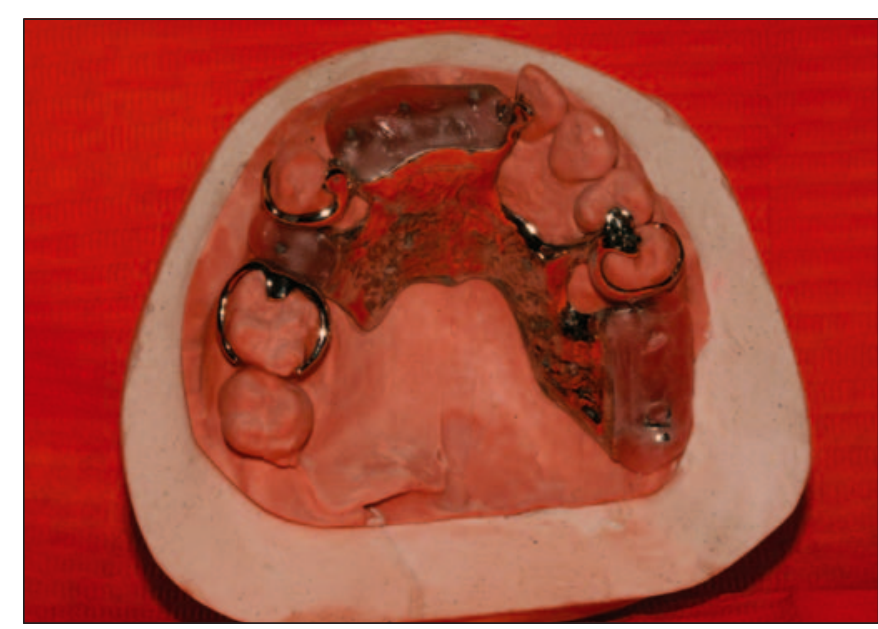

Fig. 3. Vista de los elementos que forman parte de la estructura.

dos (2). En la elaboración de las prótesis removibles, se pueden utilizar resinas termopolimerizables, aleaciones metálicas (básicamente de cromo cobalto, pese a la incorporación del titanio) y dientes acrílicos, aunque existen también materiales polímeros flexibles (Partial Flex ${ }^{\circledR}$, Valplast ${ }^{\circledR}$, Flexite ${ }^{\circledR}$ ) que aportan nuevas perspectivas a la construcción de las bases protésicas especialmente en los casos de alergias a metales (3).

Todos estos considerandos deben trasladarse al modelo de estudio y a la prescripción, que suficientemente detallada se hará llegar al laboratorio, y con posterioridad se procederá a efectuar las modificaciones dentales que resultan del proceso de análisis y diseño, como la determinación del ecuador dentario según el eje de inserción seleccionado, el tallado de planos guías y de los apoyos (4). La correcta realización de este paso es fundamental para el éxito de la prótesis. Acto seguido tomamos las impresiones definitivas con hidrocoloides irreversibles, cuando la prótesis es dentosoportada, y con pasta zinquenólica para registrar los tejidos mucosos, cuando la prótesis es mucodentosoportada. En este último caso utilizamos rodete de resina, previamente elaborado, como cubeta de registro para las zonas mucosas y también como soporte de las ceras de mordida para la relación intermaxilar. Este rodete se funcionalizará con godiva verde de baja fusión para delimitar en sus extremos las zonas móviles de frenillos y las zonas de inserción. Posteriormente raptamos con una cubeta individual el rodete, lo cual nos permitirá que 
el modelo vaciado relacione en una misma impresión los tejidos blandos, que se han tomado con el rodete y la zinquenólica, bajo una técnica funcional de presión selectiva, con los dientes registrados mediante alginato. La resultante es un modelo de trabajo que refleja el diseño y las modificaciones que se han llevado a cabo en boca. Este modelo, una vez duplicado, es el que servirá al laboratorio para la confección de la estructura metálica que será el armazón de nuestra restauración protésica.

Hay evidencias de que en una prótesis removible reciente, que no ha sufrido desajustes y está bien adaptada, no se producen reabsorciones sustanciales de las bases, al menos en los períodos iniciales, tanto si el paciente es portador de la prótesis parcial removible durante todo el día como si descansa por la noche. Ello abunda en la necesidad de mantener las bases perfectamente adaptadas para minimizar la pérdida de hueso alveolar (5). Para facilitarlo existen técnicas de rebase directo que permiten mantener la estabilidad perdida de manera simple cuando sea necesario (6).

La gran influencia del mantenimiento de las prótesis removibles en el éxito de la restauración y en la supervivencia de los dientes remanentes es lo que hace necesario establecer pautas pactadas desde el inicio. Hay muchos factores físicos, químicos, biológicos y sociales adversos en los pacientes ancianos que facilitan la aparición de caries en los dientes pilares, movilidad dental, inflamación de las mucosas y reabsorción del hueso alveolar residual. Diversos aspectos como la disminución de la capacidad masticatoria, la limitación de la autoclisis por parte de la mucosa bucal y de la musculatura lingual, la dificultad física de la habilidad manual revierten en una mayor acumulación de placa dental en las prótesis y en los tejidos circundantes. Es por ello que los dientes pilares, tienen una mayor morbilidad y son más susceptibles de padecer inflamación periodontal y/o caries.

Los portadores de prótesis removibles deben retirárselas después de cada comida teniendo la precaución de llenar la pila de agua para evitar que, si cae, pueda fracturarse. En los ancianos, la discapacidad y la disminución de la habilidad manual puede hacer necesario que sea el cuidador el que retire y limpie la prótesis y los dientes. Especialmente, dos veces al día, el paciente o el cuidador deben realizar una limpieza profunda utilizando cepillos especiales de prótesis o un cepillo de uñas y jabón. Ocasionalmente se puede complementar con un baño ultrasónico y sumergirla en un recipiente con unas gotas de hipoclorito sódico durante 20 minutos si la prótesis es de resina. Los esqueléticos pueden ver dañado el metal si se excede de los 10 minutos. En cualquier caso existen en el mercado tabletas limpiadoras que pueden cumplir perfectamente la función limpiadora si no exceden el tiempo recomendado. No deben usarse en portadores de rebase blando.

El mantenimiento, que debe formar parte desde el origen del plan de tratamiento, incluye la realización de visitas periódicas sistemáticas cada 4 o 6 meses para revisar el trauma mecánico sobre los tejidos blandos, la necesidad de rebase cuyo mejor indicador en los extremos libres es el desajuste de los retenedores indirectos, para prevenir daños en los apoyos sobre las preparaciones efectuadas para alojarlos y el posible daño en los dientes pilares. Para minimizar el riesgo y prevenir la aparición de caries en zonas de compromiso como son los pilares que sustentan la prótesis parcial removible, los pacientes deberían utilizar en el cepillado de los dientes un mínimo de 2 veces al día una pasta dental fluorada que contenga un mínimo de 1.100 ppm de flúor. Después del uso de la pasta dental fluorada, deberían usar mousse dental que contenga CPP-ACP (fosfato de calcio fosfopéptido amorfo), Recaldent ${ }^{\circledR}$ (MI Paste, GC) dos veces al día, excepto en alérgicos a la proteína de la leche, con objeto de estimular la remineralización de las superficies dentales, ya que se deposita una mayor proporción de iones fosfato y calcio en la superficie del esmalte.

Como se ha citado anteriormente, el tratamiento de elección del anciano desdentado total, en nuestra área socio-económica, es la prótesis implantorretenida (removible o fija). Pero por determinados motivos (patologías generales previas, graves alteraciones óseas maxilares, limitaciones económicas, etc.) el tratamiento indicado puede ser una prótesis completa mucosoportada (P.C.M.) (7). En una prótesis completa, se deben determinar y registrar correctamente los parámetros biológicos para que el tratamiento resulte predecible, son aquellos factores ana- 
tómicos y fisiológicos propios de cada paciente que las prótesis deben ayudar a restablecer para la recuperación de las funciones orales. Estos parámetros se muestran en la Tabla 1.

La secuencia de elaboración de la prótesis completa comienza con la toma de unos "alginatos" para obtener los modelos de estudio. Servirán para reproducir la mucosa de soporte y los tejidos blandos relacionados con los extremos protésicos y así obtener unos modelos de yeso donde confeccionar las cubetas individuales. En la arcada inferior, y por orden de trascendencia para conseguir sujeción protésica, deben reproducirse los trígonos retromolares, la inserción del músculo geniogloso, la de los músculos milohioideos, todo el fondo de vestíbulo y la cresta alveolar residual.

En la arcada superior, y siguiendo el mismo criterio de sujeción, las zonas a reproducir son las tuberosidades retromolares, el área posterior palatina (límite entre paladar blando y duro), todo el fondo de vestíbulo, la cresta alveolar residual y el paladar duro.

Se debe tener en cuenta que en un desdentado total no hay Dimensión Vertical de Oclusión (D.V.O.) (2); pero la D.V.O. suele ser 2-4 mm inferior a la Dimensión Vertical de Reposo (D.V.R.) (8) que es la longitud vertical de la cara cuando hay equilibrio entre los músculos de cierre y de apertura mandibular. Si el paciente presenta rigidez, contracturas musculares orofaciales, incomodidad o estrés, el equilibrio se verá influenciado (9). Para inducir o facilitar una posición de reposo mandibular hay técnicas que el dentista debe conocer (deglución y D.V.R., fonemas labio-labiales y D.V.R., proporciones de los tercios faciales y estética facial).

Técnicamente puede usarse silicona tipo masilla según la descripción de Zarb (10) para rellenar el espacio libre entre ambas crestas óseas desdentadas, cuando la mandíbula está en posición de D.V.O/D.V.R. Debe reproducir de forma aceptable la cresta alveolar superior e inferior y también puede usarse para determinar el soporte labial o Espacio Neutro Protésico (E.N.P.) en el sector anterior, buscando el equilibrio en la cara del paciente. Luego será posible objetivar la futura Línea Incisal (L.I.) en relación con el labio superior en reposo; y los corredores laterales o pasillos de Tomes durante la sonrisa. Con la misma mordida de silicona puede llegar a predeterminarse la posición condilar de Relación Céntrica (R.C.) si el paciente realiza un movimiento de cierre mandibular en rotación condilar antes del endurecimiento de la silicona de masilla y deteniéndose en la posición que corresponda a la futura D.V.O.

En cuanto a las referencias anatómicas, ambos trígonos retromolares deben identificarse en su totalidad y señalarse en los modelos; así como su punto medio en altura. También deben evidenciarse ambas tuberosidades retromolares, la línea de separación entre el paladar duro y blando, con la fóveas palati-

\section{TABLA 1.- PARÁMETROS BIOLÓGICOS (P.B.) DE UNA PRÓTESIS COMPLETA MUCOSOPORTADA}

\begin{tabular}{|c|c|c|c|c|}
\hline $\begin{array}{l}\text { Situación de los } \\
\text { dientes artificiales } \\
\text { en el espacio }\end{array}$ & $\begin{array}{l}\text { Propios de } \\
\text { los dientes } \\
\text { artificiales }\end{array}$ & $\begin{array}{l}\text { Propios de la } \\
\text { base protésica }\end{array}$ & $\begin{array}{l}\text { Relacionados con } \\
\text { la dinámica } \\
\text { tempormandibular }\end{array}$ & $\begin{array}{c}\text { Relacionan los } \\
\text { dientes artificiales } \\
\text { entre sí }\end{array}$ \\
\hline $\begin{array}{l}\text { D. vertical } \\
\text { L. incisal } \\
\text { P. oclusal } \\
\text { E. neutro } \\
\text { protésico }\end{array}$ & $\begin{array}{l}\text { Color } \\
\text { Tamaño } \\
\text { Forma } \\
\text { A. cuspídea } \\
\text { Material }\end{array}$ & $\begin{array}{l}\text { Forma de la } \\
\text { superficie de soporte } \\
\text { Extremos } \\
\text { Diseño (forma) } \\
\text { Material }\end{array}$ & $\begin{array}{l}\text { R. cráneo-maxilar } \\
\text { D. intercondilar } \\
\text { E. de bisagra } \\
\text { R. céntrica } \\
\text { G. condílea } \\
\text { A. de Bennet } \\
\text { M. de Bennet }\end{array}$ & $\begin{array}{l}\text { R. oclusal } \\
\text { G. incisal } \\
\text { Curvas de } \\
\text { compensación } \\
\text { Longitud posterior } \\
\text { de la arcada }\end{array}$ \\
\hline
\end{tabular}

D.: Dimensión; L.: Línea; P.: Plano; E.: Espacio ; R.: Relación; G.: Guía; A.: Ángulo. 
nas en su línea media, y la situación del futuro límite de las bases (mucosa adherida y mucosa móvil) en los fondos de saco vestibular y lingual (liberando las inserciones musculares). Para la adaptación de la mordida de silicona a los modelos de yeso deben retirarse los excesos de silicona o yeso, y con ellos se procederá al montaje de los modelos en el articulador gracias a la mordida de silicona.

Esta mordida de silicona puede seccionarse horizontalmente siguiendo una predeterminación del Plano Oclusal (P.O.) a través de la referencia clínica de la L.I. en la misma silicona y la de los trígonos retromolares en los modelos. En este P.O. (mitad inferior de la silicona sobre el modelo inferior), se puede dibujar la futura situación de las caras vestibulares de los dientes superiores; la de los anteriores según el volumen de la silicona, que daba soporte a los labios, y la de los posteriores por la relación de la cara distal de los caninos superiores con la zona externa de ambos trígonos (o gracias a los corredores bucales o pasillos de Tomes si se han determinado clínicamente).

Las cubetas individuales y planchas base con rodete serán de acrílico, auto o fotopolimerizable. La resina debe cubrir el diseño marcado en el modelo superior, y el rodete contactar con el P.O. de la silicona. Desbastando los excesos, la base superior adaptará a la futura mucosa de soporte. Su rodete de oclusión será la copia del soporte labial clínico que la silicona ofrecía con, a su vez, un P.O. de referencia según la L.I. clínica y el nivel medio de ambos trígonos. Se repite el procedimiento para la confección de la plancha base y el rodete inferior, pero ahora adaptándola al modelo inferior y al rodete superior. Con la ayuda de las cubetas individuales, deben determinarse de forma precisa los 24 parámetros biológicos clínicos para que pueda procesarse con seguridad la P.C.M. en el laboratorio. Mediante las planchas base con rodete se determina la D.V.O. según la relación de la D.V. con la deglución, los tercios faciales y la estética facial, y la articulación de los fonemas labio-labiales (D.V.R.) y dento-dentales (D.V.O.) (Fig. 4). Los rodetes, en su zona anterior, deben dar soporte labial según la estética extraoral (11). Con un buen soporte labial se localiza la L.I. superior (relacionándola con el labio superior en reposo y con el labio inferior en sonrisa), y con la D.V.O., el soporte labial y la L.I. determinados debe definirse el P.O. que debe ser paralelo al plano de Camper y a la línea bipupilar (Fig. 5) (12). Para terminar con la determinación del E.N.P., el rodete superior e inferior en sus sectores posteriores deben situarse, en sentido vestibulolingual, en equilibrio con la funcionalidad de la lengua y de las mejillas (E.N.P. en el sector posterior). El inferior no debe interferir en la dinámica lingual, y un buen diseño vestibular de las zonas posteriores y superiores favorecerá unos corredores laterales o pasillos de Tomes estéticos y funcionales. La altura del futuro central superior se plasmará en el rodete superior (labio superior en sonrisa) y también la anchura del grupo anterior (alas de la nariz y

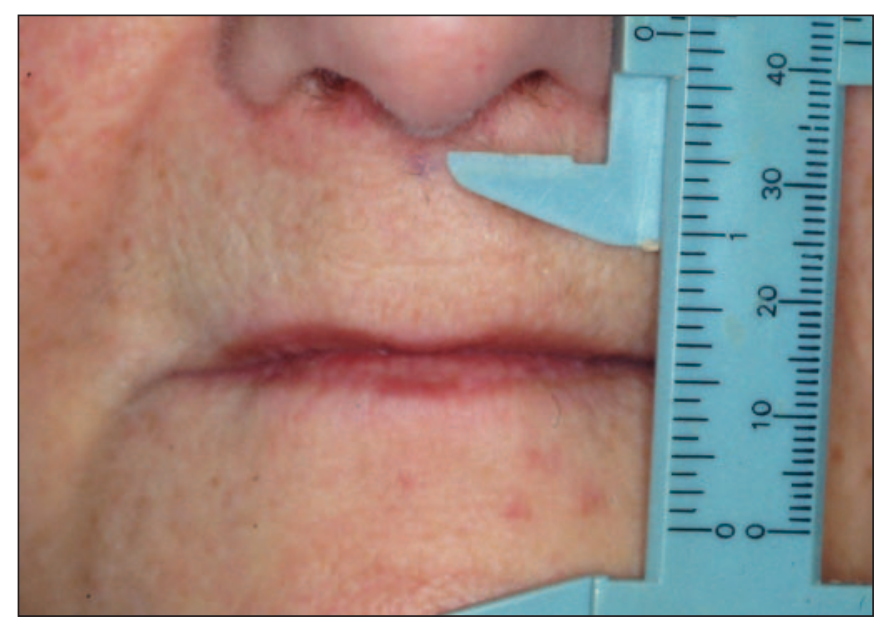

Fig. 4. Determinación de la D.V.O. con la plancha base y rodete colocada en boca.

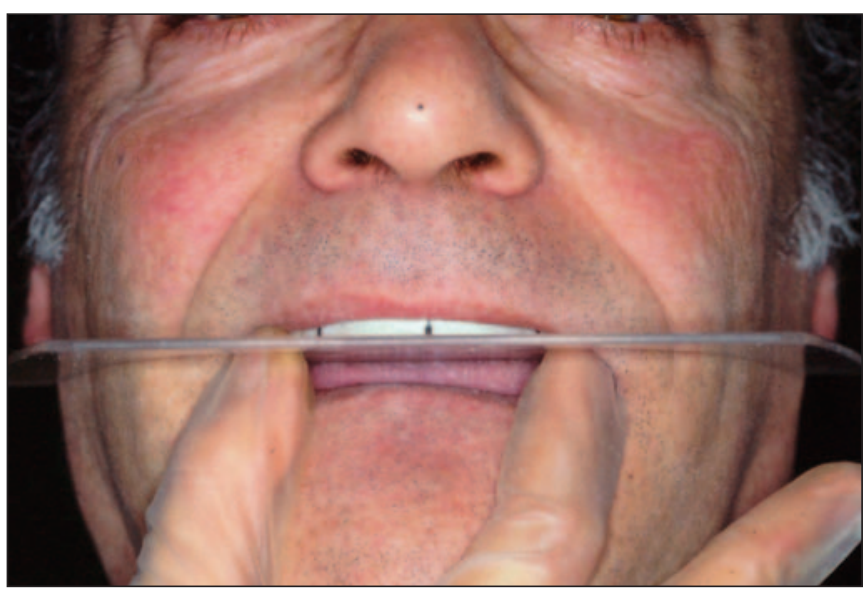

Fig. 5. Equilibrado del rodete superior para determinar el plano oclusal. 
caninos superiores) (13) (Fig. 6). Con este tipo de planchas base, para el arco facial y los registros de lateralidad y protrusiva, pueden usarse las mismas técnicas que para pacientes con dentición natural. El registro de R.C. no debe modificar la D.V.O. ni interferir con el E.N.P. En estas condiciones, se pueden tomar las impresiones definitivas, en las que la pasta zinquenólica es el material de primera elección por su precisión en situaciones de grosores mínimos. El paciente debe mantenerse en posición de R.C. mandibular y ejercer una fuerza oclusal uniforme hasta el fraguado del material.

En el vaciado de los modelos, el encofrado ayuda a reproducir las superficies de soporte y los 2-4 mm periféricos (superficie externa de la impresión y sellado periférico). El montaje en el articulador se realiza según el registro de la posición del P.O. del rodete superior (arco facial). Luego se montará el modelo inferior según el registro de R.C. que relaciona ambos rodetes. Los valores de la guía condílea y del ángulo de Bennet (y del movimiento de Bennet) se ajustarán con los registros de protrusión y lateralidad.

Los dientes artificiales se individualizan según color, anatomía vestibular, altura incisivo-gingival del incisivo central superior, tamaño mesiodistal del grupo anterosuperior, angulación cuspídea y material de los dientes artificiales (primera elección: resina acrílica) (14). Su montaje puede empezarse por una u otra arcada. Los dientes se alinean en una plancha-base y según el rodete de oclusión de la cubeta individual

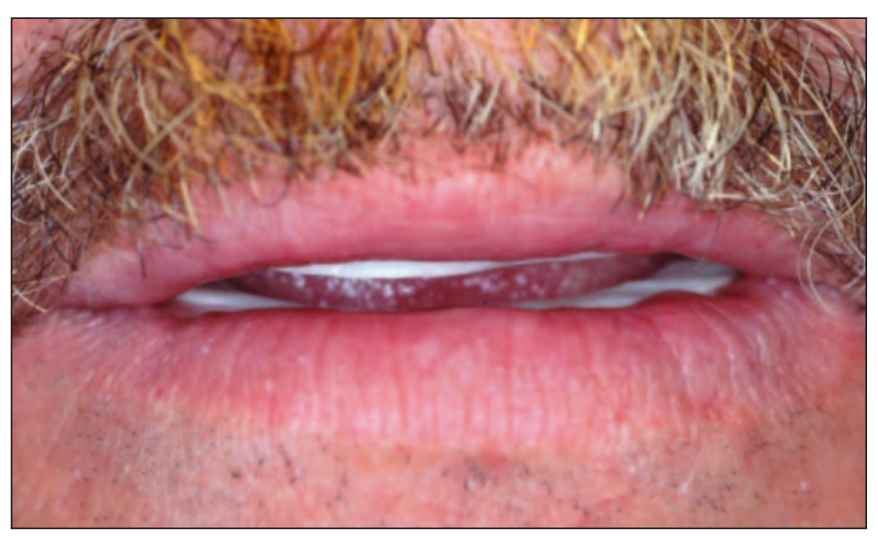

Fig. 6. Altura del rodete superior 1-2 mm por debajo del labio superior. antagonista; así se transfieren los valores clínicos a los dientes artificiales. El patrón de oclusión debe ser el de oclusión bibalanceada. Dicho montaje, se comprueba en boca para verificar todos los parámetros (color, anatomía, D.V.O., R.C., P.O., L.I., E.N.P., etc.). En este momento, es la primera ocasión en la que el paciente puede valorar "la nueva intercuspidación" por lo que hay que asegurarse de que en la posición condilar de R.C. existe una máxima intercuspidación entre los dientes posteriores y, en movimientos excéntricos, un patrón de oclusión bibalanceada (15). Dicha prueba de dientes se enmufla mediante la técnica a "cera perdida" para convertir los materiales termoplásticos en acrílicos.

Tras este paso se entrega la P.C.M. y se comprueba la estabilidad protética, sellado periférico y contactos oclusales. Existe la posibilidad de realizar ajustes y se indican las instrucciones de uso y adaptación (articulación del lenguaje, masticación, autoimagen), y de higiene y mantenimiento.

\section{PRÓTESIS SOBRE IMPLANTES}

La rehabilitación de pacientes edéntulos mediante prótesis completas mucosoportadas convencionales aumenta el grado de satisfacción del paciente, la estética y la fonética, pero la función masticatoria no suele ser del todo satisfactoria (16). La colocación de implantes osteointegrados es una opción muy interesante para instalar una prótesis fija o removible soportada en los implantes.

Una sobredentadura es una prótesis removible que se retiene mediante implantes oseointegrados, y puede tener un comportamiento biomecánico similar al de una prótesis completa convencional (soporte de las fuerzas masticatorias sobre la mucosa oral: implantorretenida pero mucosoportada) o similar al de una prótesis fija (soporte de las fuerzas masticatorias sobre los implantes: implantorretenida e implantosoportada) dependiendo del número de implantes, la distribución de los mismos por la arcada y el tipo de anclajes utilizados. En líneas generales, a mayor número de implantes, menor soporte mucoso, si la distribución de los implantes ocupa todos los sectores de la arcada (frente anterior y dos sectores posteriores) menor soporte mucoso, y a mayor rigidez de los anclajes, menor soporte mucoso. 
Frecuentemente, la falta de disponibilidad ósea para distribuir correctamente implantes en todos los sectores de la arcada es un factor determinante para optar por una sobredentadura y no por una prótesis fija, pero además dos factores comúnmente relacionados con la elección de una sobredentadura son la facilidad higiénica de la prótesis (al retirarse la prótesis se mejora el acceso a los implantes para la limpieza) y la posibilidad de eliminar la cobertura palatina de la prótesis superior, que puede dificultar la deglución, estimular el reflejo nauseoso y alterar la sensación del gusto. Sin embargo, la eliminación de la cobertura palatina de una sobredentadura superior, implica la transmisión de más carga masticatoria a los implantes remanentes (17). Otras veces, cuando ha fracasado alguno de los implantes planificados para la colocación de una prótesis fija, obliga a cambiar la opción terapéutica por una sobredentadura.

Cuando el maxilar superior es completamente edéntulo y una prótesis mucosoportada convencional no satisface las expectativas del paciente, no hay evidencia de qué tipo de prótesis sobre implantes puede mejorar la calidad de vida en general, ni el grado de satisfacción, el impacto en la salud oral, el confort, la estética, el rendimiento masticatorio, ni la fonética (18). Por tanto, el número de implantes recomendado para la colocación de una sobredentadura maxilar no está claro, pero se sabe que con menos de 4 implantes, la tasa de éxito de los implantes es significativamente inferior que con 4 o más implantes (19). Además, actualmente se considera suficiente la colocación de 6 implantes para retener una prótesis fija, de manera que con la distribución uniforme de 6 implantes repartidos por la arcada superior, el comportamiento biomecánico de una prótesis removible es similar al de una prótesis fija. Con estos datos, parece lógico pensar que para la confección de una sobredentadura en el maxilar superior necesitamos un intervalo de 4 a 6 implantes (Fig. 7). Recientemente se ha sabido que una sobredentadura superior retenida por 6 implantes y una barra ofrecen mejor grado de satisfacción a los pacientes, aunque resulta económicamente más costosa (20). También debe tenerse en cuenta que con la planificación de una sobredentadura sobre 6 implantes, la eventual pérdida de uno de ellos no suele suponer un cambio en el plan de tratamiento y es

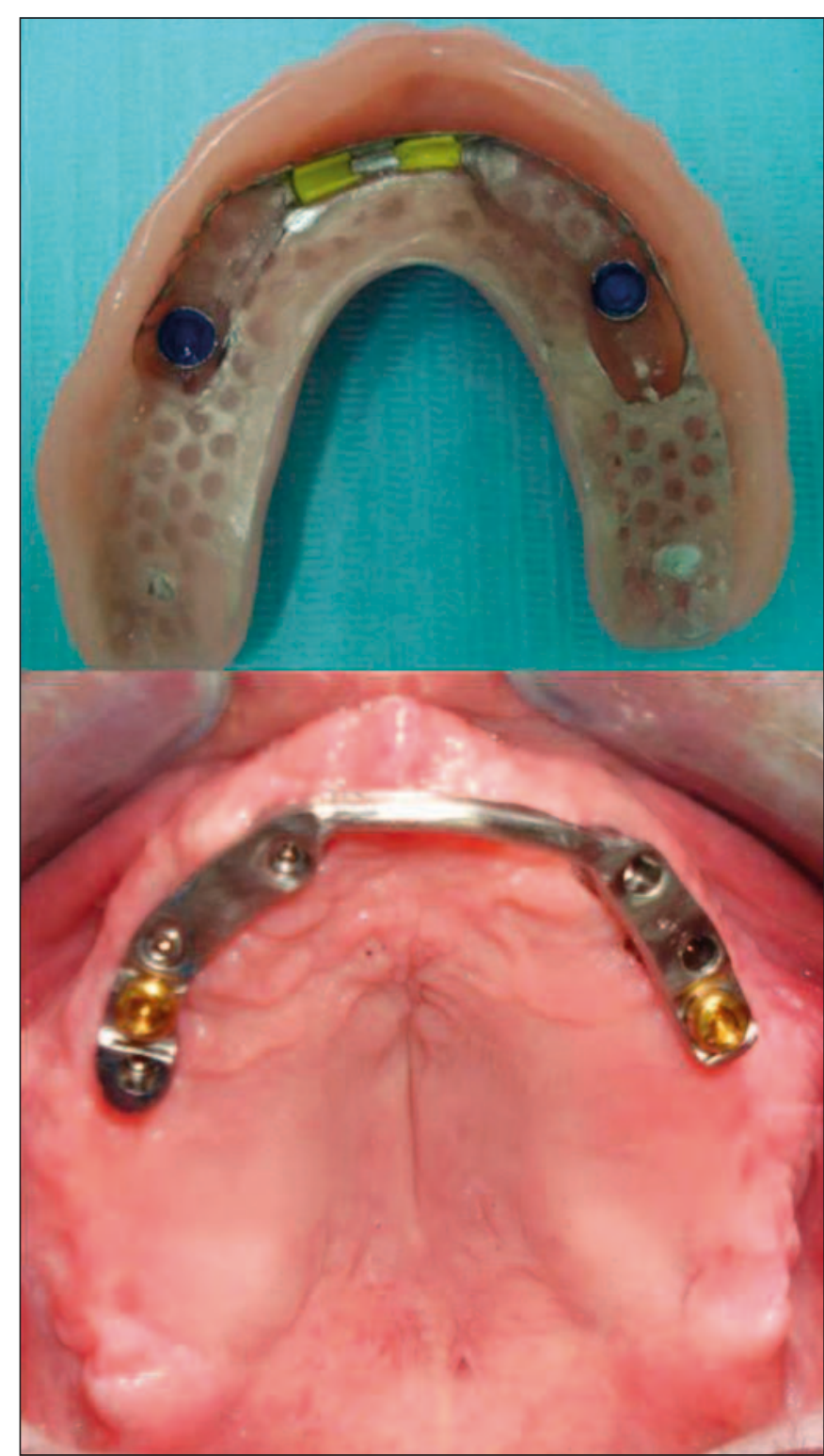

Fig. 7. Sobredentadura superior confeccionada sobre 5 implantes con diferentes anclajes.

muy probable que la misma sobredentadura pueda continuar funcionando biomecánicamente de una manera muy similar, cosa que no es posible con una planificación sobre 4 implantes (21).

En la mandíbula, en cambio, sí que existe evidencia de que los pacientes que no están satisfechos con una prótesis completa inferior convencional debido a la falta de retención y estabilidad, la opción terapéutica más favorable es la sobredentadura mediante 2 implantes (Fig. 8). Tanto es así que se consi- 
guió un documento de consenso acerca del mínimo tratamiento necesario en sobredentaduras inferiores, consistente en la colocación de 2 implantes y una sobredentadura, independientemente del sistema de anclaje utilizado (22). Es la opción que permite a los pacientes sentir la prótesis más confortable, estable, y mejora su capacidad masticatoria y fonética.

Existen diferentes sistemas de anclaje para retener una sobredentadura (bolas, barras, Locators ${ }^{\circledR}$, coronas telescópicas, imanes, etc.). De entre ellos, el anclaje de tipo Locator ${ }^{\circledR}$ ha demostrado unas prestaciones igual o superiores a los demás en cuanto a complicaciones a largo plazo y mantenimiento de las funciones orales (23), y además es el preferido por los pacientes y profesionales en cuanto a la facilidad para mantenerlo en buenas condiciones higiénicas (24).

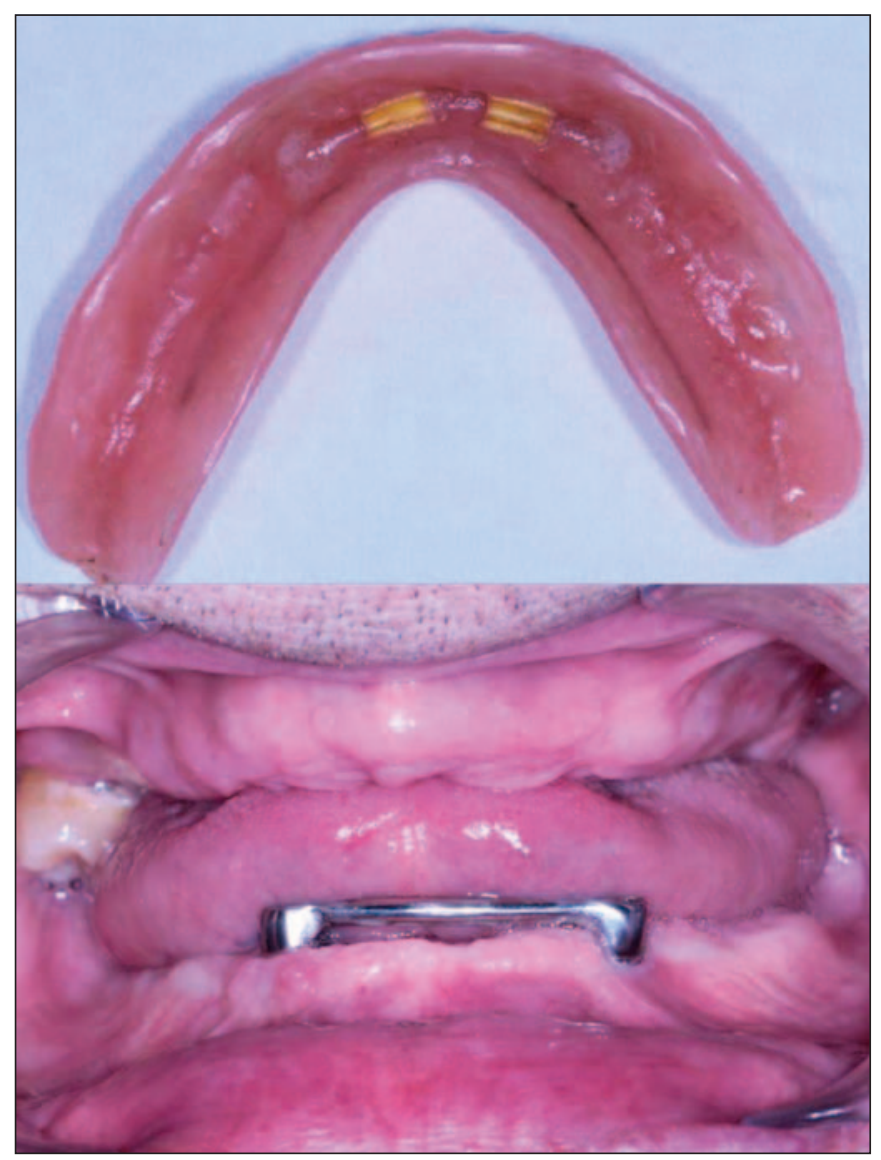

Fig. 8. Sobredentadura inferior confeccionada sobre 2 implantes, barra y caballetes.
Conviene considerar que todo diseño (incluyendo la distribución de los implantes y el funcionamiento de los anclajes) que permita un eje de rotación anteroposterior de la sobredentadura respecto de los anclajes, ayudará a que la prótesis sea mucosoportada y los implantes sirvan como sistema de retención. Por ejemplo, 2 implantes intermentonianos con 2 anclajes tipo Locator ${ }^{\circledR}$ o ferulizados por una barra cilíndrica permiten dicha rotación antero-posterior. En cambio, cuando existen más de 2 implantes y se generan más puntos de apoyo, se elimina este eje de fulcro y la biomecánica de la prótesis empieza a semejar la de una prótesis fija, y esto es sumamente importante a la hora de pensar en el número de implantes necesario para la rehabilitación que necesita el paciente.

En cuanto a la ferulización o no de los implantes, parece ser que los anclajes axiales tipo Locator ${ }^{\circledR} \mathrm{o}$ bolas $\mathrm{O}$-ring ${ }^{\circledR}$ requieren de un mayor mantenimiento, puesto que las hembras de goma o teflón sufren un deterioro más frecuente. Por otro lado los anclajes de barra presentan peores condiciones higiénicas, y frecuentemente los pacientes sufren la hipertrofia de la mucosa periimplantaria y aumentan los índices de sangrado. Por estos motivos, aunque el tipo de anclaje no tiene un efecto notorio en el grado de satisfacción de los pacientes portadores de sobredentaduras, y tampoco hay diferencias significativas en cuando a las tasas de supervivencia de los implantes ferulizados o no ferulizados, la capacidad higiénica del paciente es un factor clave en la elección del tipo de anclaje (25).

Existen en la actualidad numerosos sistemas digitales de diseño y fabricación de prótesis, los llamados sistemas de CAD-CAM. Sus aplicaciones en cuanto a las prótesis removibles sobre implantes son importantes, ya que dichas técnicas nos permiten planificar la prótesis y controlar muchos de los parámetros (volúmenes y espesores de los diferentes materiales que componen la prótesis, resistencia de los mismos) anteriormente a la confección de la misma (Fig. 9), y conseguir mejores ajustes en menor tiempo (1). Probablemente, esta será la manera que dispongamos en un futuro cercano de minimizar complicaciones y facilitar el mantenimiento de los pacientes portadores de prótesis removible sobre implantes, especialmente aquellos de edad avanzada y con cierta dependencia en su rutina diaria. 


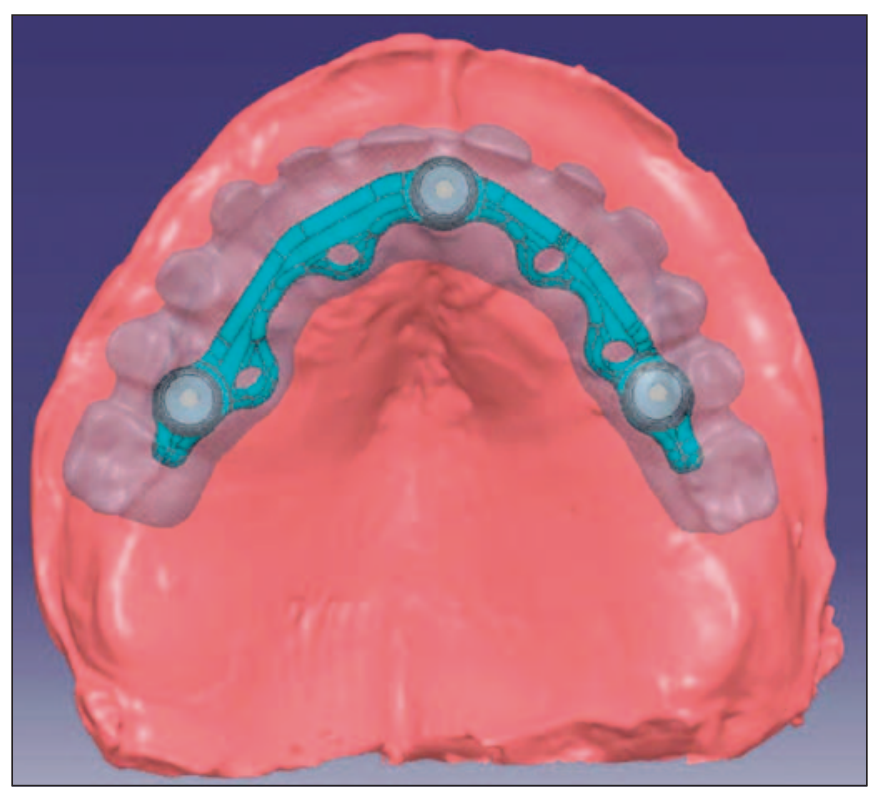

Fig. 9. Estudio de espesores y volúmenes previo a la fabricación de una sobredentadura por cad-cam.

\section{BIBLIOGRAFÍA}

1. Lang LA, Tulunoglu I. A critically appraised topic review of computer-aided design/computer-aided machining of removable partial denture frameworks. Dent Clin North Am 2014;58:247-55.

2. Sha R, Aras M. Esthetics in Removable partial denture. A review. Kathmandu Univ Med J 2013; 44:344-8.

3. Fueji K, Ohkubo C, Yatabe M. Clinical application of removable partial dentures using thermoplastic resin-part I: definition and indication of non-metal clasp dentures. J Prosthodont Res 2014;58:3-10.

4. Mallat E. Prótesis Parcial Removible y sobredentaduras. Elsevier: Madrid; 2004.

5. Kovačević Pavičić D, Lajnert V, Simonić Kocijan S, Uhač I, Glavičić S, Kovač Z. The effect of frequent removable partial denture wearing on alveolar bone resorption. Med Glas (Zenica) 2013; 10:373-8.

6. Hill E, Rubel Barry. Direct chairside had reline at delivery of a newly fabricated distal extension removable partial denture: Considerations and techniques. J Can Dent Assoc 2011;77:b84.

7. Misch C. Implantología Contemporánea. Barcelona. Elsevier: Barcelona, 2009.

8. Mays KA. Reestablishing occlusal vertical dimension using a diagnostic treatment prosthesis in the edentulous patient: a clinical report. J Prosthodont 2003;12:30-6.

9. Casselli H, Landulpho AB, Silva WA, Silva FA. Electrognathographic evaluations of rehabilitated edentulous patients. Braz Oral Res 2007;21:35561.

10. Zarb G, Bolender C, Hickey J, Carlsson G. Boucher's Prosthodontic Treatement for Edentulous Patients. Mosby Company: St. Louis; 1990.

11. Sadowsky SJ. The role of complete denture principles in implant prosthodontics. J Calif Dent Assoc 2003;31:905-9.

12. Nissan J, Barnea E, Zeltzer C, Cardash HS. Relationship between occlusal plane determinants and craniofacial structures. J Oral Rehabil 2003; 30:587-91.

13. Coachman C, et al. Smile Design: From Digital Treatment Planning to Clinical Reality. http:/www. simposiointernacional.com.ec/files/Interdisciplinary \%20Treat\%20Planning\%20Chapter.pdf. Accedido en 12 de marzo de 2015.

14. Valiati F, Belser U. Full-mouth adhesive rehabilitation of a severely eroded dentition: the threestep technique. Part 1. Eur J Esthet Dent 2008;3: 30-44.

15. Klineberg I, Kingston D, Murray G. The bases for using a particular occlusal design in tooth and implant-borne reconstructions and complete dentures. Clin Oral Implants Res 2007; 18 (Suppl 3):189-92.

16. Awad MA, Lund JP, Shapiro SH, Locker D, Klemetti E, Chehade A, et al. Oral health status and treatment satisfaction with mandibular 
implant overdentures and conventional dentures: a randomized clinical trial in a senior population. Int J Prosthodont 2003;16:390-6.

17. Ochiai K, Williams B, Hojo S, Nishimura R, Caputo AA. Photoelastic analysis of the effect of palatal support on various implant-supported overdenture designs. J Prosthet Dent 2004;91: 421-7.

18. Slot W, Raghoebar GM, Vissink A, Huddleston Slater JJ, Meijer HJA. A systematic review of implant-supported maxillary overdentures after a mean observation period of at least 1 year. J Clin Periodontol 2010;37:98-110.

19. Kern JS, Kern T, Wolfart S, Heussen N. A systematic review and meta-analysis of removable and fixed implant-supported prostheses in edentulous jaws: post-loading implant loss. Clin Oral Impl Res 2015;00:1-22.

20. Listl S, Fisher L, Giannakopoulos NN. An economic evaluation of maxillary implant overdentures based on six vs. four implants. BMC Oral Health 2014; 14:105-14.

21. Raghoebar G, Meijer H, Slot W, Huddleston Slater JJ, Vissink A. A systematic review of implantsupported overdentures in the dentulous maxilla, compared to the mandible: How many implants? Eur J Oral Implantol 2014;7(Suppl):1-11.
22. Feine JS, Carlsson GE, Awad MA, Chehade A, Duncan WJ, Gizani S, et al. The McGill Consensus Statement on Overdentures. Montreal, Quebec, Canada. May 24-25, 2002. Int J Prosthodont 2002; 15:413-4.

23. Roccuzzo M, Bonino F, Gaudioso L, Zwahlen M, Meijer HJA. What is the optimal number of implants for removable reconstructions? A systematic review on implant-supported overdentures. Clin Oral Implants Res 2012;23(Suppl.6):229-37.

24. Cordaro L, Mirisola di Torresanto V, Petricevic N, Roig Jornet P, Torsello F. Single unit attachments improve peri-implant soft tissues conditions in mandibular overdentures supported by four implants. Clin Oral Impl Res 2013;24:536-42.

25. Stoumpis C, Kohal RJ. To splint or not to splint oral implants in the implant-supported overdenture therapy? A systematic literature review. J Oral Rehabil 2011;38:856-69.

\section{CORRESPONDENCIA}

Dr. Manuel Ribera Uribe

Universitat Internacional de Catalunya

Josep Trueta, s/n

08195 Sant Cugat del Vallés

Correo electrónico: mribera@uic.es 